\title{
Ungewöhnliche Verwendungen von mit (II) ${ }^{1}$
}

\section{Die Ellipsen-Lösung}

Das Semi-Satzglied mit (eine spezielle Form des Verbgruppenadverbiales) erfüllt nicht die Bedingungen, die kategorial an die Füllung einer solchen Satzgliedstelle zu stellen sind, insofern als es keine vollständige Phrase darstellt. Welche Beschreibungsmöglichkeiten bieten sich? Ich erörtere zunächst die Möglichkeit einer Erklärung durch Ellipse einer NP oder eines Pronomens (vgl. auch Klein 1993; Pasch 1995; Wilder 1996). Bei Präpositionen ist im allgemeinen die Ellipse der regierten Phrase nur in sog. Vorwärtsellipsen bei Koordination möglich (die Position der elidierten Phrase wird durch [] gekennzeichnet):

(21) Kühe standen neben [ ] und hinter dem Stall.

(22) Elisabeth kam vor [ ], Hans jedoch erst nach dem Mittagessen.

Es ist also hier zur Restitution der Ellipse jeweils vorzugreifen auf die NP dem Stall bzw. dem Mittagessen, die auf das Vorkommen der koordinierten zweiten Präposition unmittelbar folgt. Man vergleiche demgegenüber die nichtgrammatische Rückwärtsellipse.

(21a) *Kühe standen neben dem Stall und hinter [ ].

(22a) *Elisabeth kam vor dem Mittagessen, Hans jedoch erst nach [ ].

Nur bei dem Präpositionenpaar mit/ohne ist eine solche Rückwärtsellipse neben der Vorwärtsellipse möglich:

Vorwärtsellipse:

(23) Hans arbeitete mit [ ], Fritz ohne Mundschutz. Rückwärtsellipse:

(23a) Hans arbeitete mit Mundschutz, Fritz ohne [ ]. Auch wenn mit (ähnlich wie ohne) somit bereits eine Art „Ellipsenvorsprung“ gegenüber anderen Präpositionen aufweist, so ist doch zu bedenken, daß es sich hier um rein sprachlich gestützte Ellipsen handelt, d.h., das fehlende Material kann Wort für Wort aus dem unmittelbaren Satzkontext ergänzt werden. Für diese Art der Ellipse ist auch der Doppelbegriff „Ana-/ Katalepse" eingeführt worden (vgl. Zifonun / Hoffmann / Strecker u. a. 1996, Kap. H2).

Bei Ellipsen in unserem Problemfall kann jedoch meistens nicht auf im unmittelbaren Satz- kontext vorkommende Ausdrücke vor- oder zurückgegriffen werden. Vielmehr muß häufig, wenn wir überhaupt eine Ellipsenlösung anstreben, die fehlende Stelle aus dem weiteren Kontext oder aus dem Wissen ergänzt werden oder aber auch indefinit besetzt werden. So ist etwa bei (5)

(5) ... die Liberalen oder Sozialdemokraten, die mit am Gebäude des Staates zimmern

aus dem Wissen die Ergänzung mit den Christdemokraten möglich.

Der Standardwert einer indefiniten Besetzung ist mit anderem/mit anderen, man vergleiche etwa:

(12) weil der saure Regen mit eine Ursache für das Waldsterben ist

(12b) weil der saure Regen mit anderem eine Ursache für das Waldsterben ist

Eine definite Besetzung aus dem weiteren Kontext könnte etwa bei (24) vorliegen. (Die definite Bezüglichkeit ist durch den Index angedeutet.)

(24) Heinrich verließ am nächsten Tag die Stadt. Luise reiste mit.

(24a) Heinrich ${ }_{i}$ verließ am nächsten Tag die Stadt. Luise reiste mit $\mathrm{ihm}_{\mathrm{i}}$.

Innerhalb des unmittelbaren Satzkontextes haben wir es mit einer reflexiven Ellipse zu tun, vgl. (6):

(6) Ihr Dackel „Samba“, den sie meistens mit auf Reisen nimmt, ...

(6a) Ihr Dackel „Samba“, den sie meistens mit sich auf Reisen nimmt, ...

Eine Ellipsen-Lösung im Sinne der indefiniten Ellipse sieht die Grammatik von K. F. Becker (1870) vor, die als einzige deutsche Grammatik überhaupt, soweit ich das übersehe, das nichtregierende mit behandelt.

Bevor ich die Bedingungen der genannten Belegungsmöglichkeiten weiter analysiere, möchte ich zunächst auf den theoretischen Aspekt dieser Lösung etwas genauer eingehen.

Wenn wir mit als Präposition betrachten, die die definite, indefinite und reflexive Restituierung einer elidierten NP zuläßt, so drängen sich

\footnotetext{
1 Der erste Teil dieses Beitrags erschien in DaF 4/1996, 218-222.
} 
Parallelen mit der fakultativen Valenz beim Verb auf. Ganz analog zu der vorliegenden Verwendung spricht etwa Sæbø (1984: 99) von ,indefinit- und definitfakultativer Valenz" beim Verb. „Im Prinzip ist es entweder eine durch den Existenzquantor gebundene oder eine freie Variable, die unterstellt oder mitverstanden wird." Beispiele sind etwa:

indefinitfakultativ:

(25) Was tun wir aber mit all diesen Kleidern? Wir können sie ja verschenken. (ergänzen: an irgendjemanden)

definitfakultativ:

(26) Zum Teufel mit der Perle, ich ziehe die gesunde Auster vor. (ergänzen: ihr)

Definite Fakultativität verläuft bei mit in direkter Übereinstimmung zum entsprechenden Fall beim Verb. Bei indefiniter Fakultativität schlage ich bei mit die Belegung durch das Indefinitum anderer vor, die hier einer Belegung durch irgendein aufgrund der speziellen Bedeutung von mit wohl vorzuziehen ist. Reflexive Fakultativität allerdings findet beim Verb keine systematische Entsprechung. Dies wird noch zu erklären sein.

Üblicherweise spricht man im Zusammenhang mit Präpositionen nicht von Valenz, sondern einfach von Rektion. Die Valenzbegrifflichkeit wähle ich jedoch aus zwei Gründen. Zum einen, wie bereits angedeutet, ist das Phänomen der Fakultativität immer im Valenzkontext diskutiert worden, nicht im Zusammenhang mit Rektion. Zum anderen aber möchte ich hier neben der formbezogen syntaktischen Valenzbeziehung auch von einer semantischen Valenzrelation Gebrauch machen. Ein semantisches Korrelat des allgemeinen Rektionsbegriffs liegt sehr viel weniger auf der Hand. Präpositionen werden häufig, vor allem z. B. bei Eroms (1981), semantisch als zweistellige Prädikate interpretiert, wobei das eine der beiden Argumente - ich möchte es das ,innere“ nennen durch die von der Präposition regierte Phrase vertreten wird, während das andere Argument, das „äußere“, gar nicht im unmittelbaren Kontext der Präposition erscheint, sondern aus dem Satz erschlossen werden muß. Im einfachsten Fall kann das äußere Argument durch das Subjekt eines entsprechenden Kopulasatzes vertreten sein:

(27) Das Buch ist auf dem Tisch.

äußeres Arg inneres Arg
Ich möchte der Frage, wo sonst das äußere Argument bei Präpositionen ganz allgemein noch zu finden sein kann, hier nicht weiter nachgehen. Halten wir also fest, daß die Präposition mit als ein zweistelliges Prädikat , $\mathrm{x}$ mit $\mathrm{y}^{\text {‘ ver- }}$ standen werden kann. Für die Präposition mit scheint nun zumindest zu gelten, daß sowohl das Subjekt als auch das Objekt eines Satzes das äußere Argument von mit vertreten kann. Man vergleiche:

(28) Hans geht mit Elise zum Zahnarzt.

,x mit $\mathrm{y}^{\mathrm{c}}$

,Hans mit Elise

(29) Friedrich schickt Hans mit Elise zum Zahnarzt.

,x mit $\mathrm{y}^{\mathrm{x}}$

,Hans mit Elise

Das nicht-regierende mit hat kein regiertes Komplement, das innere Argument wird syntaktisch nicht ausgedrückt. Über die semantische Belegung des inneres Arguments y wissen wir bereits, daß sie in unserem elliptischen mitGebrauch drei Formen annehmen kann: die indefinite, die definite und die reflexive Ellipse. Definite und indefinite Ellipse sind beides satzübergreifende Phänomene. Die Restitution erfolgt nicht im unmittelbaren Satzkontext. Wir fassen daher beide Fälle zusammen unter dem Typ „satzübergreifende Restitution“. Die reflexive Ellipse dagegen konstituiert den Typ „satzinterne Restitution“. Unter Restitution haben wir dann generell eine semantische Wiederherstellungsprozedur mit der Möglichkeit der Verbalisierung des restituierten Elements als inneres Argument von mit zu verstehen. Im Hinblick auf die oben bereits angeschnittene Frage, warum reflexive Ellipse bei Verbvalenz keine systematische Rolle spielt, können wir nun argumentieren: Die durch Präpositionen ausgedrückten Prädikationen sind sekundär eingebettet in Satzstrukturen mit verbalem Prädikat; ihr äußeres Argument wird sozusagen an die verbale Struktur abgetreten. Dadurch wird jedoch - im Gegenzug - die Möglichkeit eröffnet, daß Komplemente des verbalen Prädikats, vor allem das Subjekt, als Bezugsausdrücke für einen reflexiven satzinternen Rückbezug des inneren Arguments der Präposition dienen können.

Wir haben nun alle „Zutaten“ für eine Klassifikation. Wir unterscheiden auf der ersten Stufe zwischen satzübergreifender und satzinterner 
Restitution und auf der zweiten Stufe zwischen der Belegung des äußeren Arguments durch das Subjekt oder das Objekt:

[1] satzübergreifende Restitution, y (inneres Argument) definit / indefinit elliptisch

[1.1] x (äußeres Argument) = Satzsubjekt

(28a) Hans geht mit zum Zahnarzt.

(30) Hans trägt die Verantwortung mit.

(31) Viele waren an der Vorbereitung zum 25. Jahrestag beteiligt. Hans bereitete die Veranstaltung mit vor.

(32) Hauptzeuge gegen Friedrich M. war dessen jüngerer Bruder. Aber auch der heutige Zeuge brachte Friedrich M. mit in Verdacht.

[1.2] x (äußeres Argument) = Satzobjekt

(29a) Friedrich schickt Hans mit zum Zahnarzt.

(33) Eva packt den Kuchen mit in das Paket.

(34) Die Polizei sperrte den Komplizen mit ein.

(35) Hans hatte eine Menge organisatorische Dinge zu erledigen. So bereitete er die Veranstaltung mit vor.

(36) Hauptbeschuldigter ist nach wie vor Egon S. Aber der heutige Zeuge brachte auch Friedrich M. mit in Verdacht.

[2] satzinterne Restitution, y (inneres Argument) reflexiv elliptisch, Bezug: Satzsubjekt

$\mathrm{x}$ (äußeres Argument) = Satzobjekt

(37) Hans nimmt Elise mit zum Zahnarzt.

(38) Eva hat den Hund mit.

(39) Eva bringt den Hund mit.

Die Klassifikation ist zu kommentieren:

a) Bestimmte Verwendungen von mit lassen bei manchen Verben Analysen nach [1.1] und nach [1.2] zu, also sowohl eine „Orientierung" am Subjekt wie auch am Akkusativobjekt. Ich habe dies durch die unterschiedlichen Kontexte bei mit vorbereiten und mit in Verdacht bringen zu verdeutlichen versucht. Die Bezugsmöglichkeit sowohl auf das Subjekt als auch auf das Objekt ist, wie bereits erwähnt, eine typische Eigenschaft (einer bestimmten Untergruppe) von Verbgruppenadverbialia.

Dagegen scheint eine Orientierung an Dativ-, Genitiv- oder Präpositivkomplement ausgeschlossen zu sein. Auch dies ist eine generelle Eigenschaft von Adverbialia. Sie steht im Zusammenhang mit der Tatsache, daß Subjekte und in zweiter Linie direkte Objekte als Bezugsgrößen grammatischer Verfahren, etwa bei der Diathesenbildung, der Reflexivierung und der Orientierung im
Sinne von Bech (1983), gegenüber anderen syntaktischen Funktionen präferiert werden (vgl. dazu etwa Primus 1987).

Der Bezug wird in erster Linie semantisch oder semantisch-pragmatisch gesteuert. Als topologische Bedingung für eine Bezugsmöglichkeit gilt jedoch, daß mit auf die Bezugskonstituente folgen muß. D. h., in dem Satz

(35a) Hans bereitete mit die Veranstaltung vor.

ist nur Hans als äußeres Argument möglich, nicht die Veranstaltung, während bei

(35b) Hans bereitete die Veranstaltung mit vor.

beide Interpretationen möglich sind.

b) Bei den Transportverben ist zu unterscheiden zwischen der Gruppe nehmen, tragen, bringen usw. einerseits und der Gruppe schicken, werfen, gießen usw. andererseits. Bei der ersten Gruppe macht das Agens selbst die Ortsveränderung mit; bei der zweiten bleibt es stationär, und nur das Patiens erfährt eine Ortsveränderung. Nur die erste Gruppe läßt die elliptische Restitution mit Bezug auf das agentische Subjekt zu (Typ [2]), während die zweite Gruppe dies ausschließt. Unbeschadet dieser Unterscheidung kann gegebenenfalls bei Verben aus beiden Gruppen eine satzexterne Restitution mit beiden Bezugsmöglichkeiten erfolgen:

[1.1]

(40) Zwei starke Männer versuchten, das Klavier hochzuschleppen, schafften es aber nicht. Hans trug dann das Klavier mit die Treppe hinauf.

[1.2]

(41) Die Möbelpacker hatten sich einige Gegenstände zum Hinauftragen bereitgestellt. Das Klavier trugen sie gleich mit hinauf.

[2]

(42) Wo Hans auch hingeht, er trägt immer einen schweren Rucksack mit.

[1.1]

(43) Zwei starke Männer versuchten, den schweren Bottich auszugießen. Hans goß dann auch mit aus.

\section{[1.2]}

(44) Die Männer hatten sich vorgenommen, die Bottiche zum Schluß auszugießen. Das Faß in der Ecke gossen sie dann auch mit aus.

[2]

(45) *Sie gossen die Bottiche mit [sich] aus.

c) Die Klassifikation erfaßt bestimmte Fälle 
nicht, bei denen nur eine Teilbedingung der internen Restitution erfüllt ist, nämlich Fälle, in denen wie bei [2] beide Argumente von mit semantisch aus dem unmittelbaren Satzkontext rekonstruiert werden können, bei denen aber eine reflexive Verbalisierung nicht möglich ist. Wir sprechen hier im Gegensatz zur Restitution von der Rekonstruktion des inneren Argumentes. Restitution ist Rekonstruktion plus mögliche Verbalisierung, Rekonstruktion Restitution minus mögliche Verbalisierung. Es handelt sich um Beispiele mit dreiwertigen Transaktionsverben:

(46) Eva gibt dem Gast ein Stück Kuchen mit.

y (inneres Argument) keine reflexive Ellipse möglich, Bezug: Dativobjekt

x (äußeres Argument) = Satzobjekt

,der Kuchen ist mit dem Gast"

(46a) *Eva gibt dem Gast ein Stück Kuchen mit sich.

ähnlich auch mit Bezug auf das Satzsubjekt als inneres Argument:

(47) Hans bringt dem kleinen Jungen ein Spielzeug mit.

(47a) ${ }^{\bar{*} \mathrm{H}}$ ans bringt dem kleinen Jungen ein Spielzeug mit sich.

Reflexivierungen mit Bezug auf einen Dativ werden häufig als ungrammatisch eingeordnet. Dem ist jedoch nicht zuzustimmen (vgl. Eisenberg 1994: 192).

Ist also das „Reflexivierungsverbot“ hier rein lexikalisch zu erklären? Steht es im $\mathrm{Zu}$ sammenhang mit der oben geschilderten Sonderentwicklung von Bewegungs- und Transportverben, bei denen bevorzugt mit an das Verb in der rechten Klammer herangerückt wird, so daß es den Status eines abtrennbaren Verbpräfixes erlangt? Man beachte jedoch, daß auch die Variante mit ins Mittelfeld vorgerücktem mit durchaus möglich ist:

(46b) daß Eva dem Gast ein Stück Kuchen mit auf die Reise gibt und

(47b) daß Hans dem kleinen Jungen ein Spielzeug mit nach Hause brachte

Die Gründe für das Reflexivierungsverbot sind somit nicht klar. Ich habe jedoch den Eindruck, daß die Restitution einer reflexiven Ellipse tendenziell bereits beim zweiwertigen Verb zu wenig akzeptablen Ergebnissen führt und eher den Status einer metasprachlichen Rekonstruktion hat. Im dreiwertigen Fall werden die Umschreibungen vollends unakzeptabel, die elaborierte Struktur unterbindet eine hyperexplizite Verbalisierung satzinterner Bezüge. Es gibt also gute Gründe, die Ellipsenlösung nicht vorschnell zu akzeptieren, sondern zunächst die andere mögliche Lösung, die Kategorisierung von nicht-regierendem mit als Adverb, einer Prüfung zu unterziehen.

\section{Die Adverb-Lösung}

Die Adverb-Lösung für das nicht-regierende mit wird in den großen deutschen Wörterbüchern vertreten. Sie grenzen das Adverb vom abtrennbaren Verbpräfix unisono durch eine semantische Bestimmung ab: Das Adverb bezeichne im Gegensatz zur Partikel eine vorübergehende Beteiligung am vom Verb denotierten Vorgang. Diese Beschreibung ist satzsemantisch zu unpräzise, um tragfähig zu sein. Bedeutet sie, daß bei Er will mit arbeiten. der mit er Gemeinte zu arbeiten aufhört, während die anderen noch arbeiten, im Gegensatz zu Er will mitarbeiten., wo alle gleichzeitig an der Arbeit sind? Ich meine, so ist der Unterschied nicht zu fassen. Mit dem syntaktischen Aspekt der Adverbkategorisierung befassen sich die Wörterbücher nicht.

Vergleichen wir zunächst am Beispiel des Paares unter (Präposition) und unten (Adverb) die Argumentstrukturen:

(48) Ich sehe Eva unter der Palme. ,Eva unter der Palme،

(49) Ich sehe Eva unten. ,Eva unten“

Die Präposition drückt eine zweistellige Relation aus, das Adverb - in bestimmten Fällen eine einstellige Prädikation. Das innere Argument der Präposition ist syntaktisch und semantisch explizit; das Adverb hat weder syntaktisch noch semantisch ein inneres Argument; man kann es allenfalls als inkorporiert betrachten. $\mathrm{Zu}$ erinnern ist daran, daß im Nhd. lokales Adverb und lokale Präposition in aller Regel nicht gleich lauten; nur die selbst bereits auf adjektivische oder nominale Bestandteile zurückgehenden rechts, links, diesseits, jenseits haben eine Verwendung als Adverb und als Präposition. Analysieren wir mit im regierenden und nichtregierenden Fall nach Maßgabe eines Paars aus Präposition und (zugehörigem) Adverb, so ergibt sich z. B. bei (28) oben:

(28) Hans geht mit Elise zum Zahnarzt. ,Hans mit Elise‘ 
(28a) Hans geht mit zum Zahnarzt. ,Hans mit ${ }^{*}$

Bei dieser Analyse von nicht-regierendem mit als Adverb ist weder Restitution noch Rekonstruktion eines inneren Arguments erlaubt. D. h., auch diese Analyse wird nicht-regierendem mit nicht wirklich gerecht. Wir haben bei der ausführlichen Diskussion in Abschnitt 3 festgestellt, daß mit zumindest die (semantische) Rekonstruktion, häufig aber auch die syntaktische Restitution des inneren Arguments erlaubt (wobei Restitution Rekonstruktion impliziert, das Umgekehrte jedoch nicht gilt). Mit steht also zwischen den beiden Extrempositionen der Kategorisierung als Präposition (unter Ellipse und Restitution des inneren Arguments) und der Kategorisierung als Adverb (unter vollständiger Absorption des inneren Arguments ohne Restitution und Rekonstruktion). Bei abtrennbaren Verbpräfixen ist das innere Argument nur rekonstruierbar, nicht restituierbar. Restituiert man es, so wird die Präfix-Verb-Bindung aufgelöst und die Partikel als Präposition reanalysiert:

(50) Hans schob ein Brett unter. ,ein Brett unter etwas'

(Rekonstruktion)

(50a) Hans schob ein Brett unter etwas.

(Restitution, Reanalyse der Partikel)

Ausgeklammert wurde bisher das Vorkommen von mit beim Superlativ (vgl. Beispiel (2)). Diese Verwendung scheint dem Adverbgebrauch am nächsten zu stehen. Die Semantik des Superlativs schließt eine wörtliche Interpretation unter Rekonstruktion des inneren Arguments aus: Der Superlativ zeichnet jeweils eine bestimmte Größe, das Denotat des Arguments der Prädikation, als diejenige aus, die als einzige den höchsten Grad der graduierbaren Eigenschaft erfüllt. Die wörtliche Interpretation von mit als ,mit anderen " widerspricht dieser Einzigkeitsbedingung. Mit erscheint hier als Superlativmodifikator, der bei einem graduierbaren Adjektiv X eine Herabstufung der Einzigkeitsbedingung $\mathrm{zu}$ einer Inklusionsbeziehung etwa im Sinne von ,zu den X-sten gehörig' ausdrückt. Mit ist der einzige Superlativmodifikator; man beachte, daß er bei attributivem Gebrauch des Superlativs syntaktisch auf der ganzen NP operiert:

(51) Das ist mit die beste Idee, die ich je von dir gehört habe.

(52) Mit die besten Brötchen bäckt in dieser Stadt $\overline{\text { die }}$ Bäckerei Lutz.
Eine weitere syntaktische Analyse dieses mit sei hier ausgeklammert.

Die kurze Diskussion der Verwendung von mit beim Superlativ hat nun gezeigt, daß nichtregierendes mit in der Tat die gesamte Bandbreite des Zugriffs auf ein inneres Argument bietet, die beim Übergang von der Präposition zum Adverb zu erwarten ist: syntaktische Restitution, semantische Rekonstruktion und Absorption ( $=$ mangelnde semantische Rekonstruktion). Es zeigt sich somit, daß keine der beiden Lösungen, die elliptische und die adverbiale, adäquat ist, insofern jede das im Übergang befindliche mit auf die diskreten Endpunkte einer Skala festlegt. Während das abtrennbare Verbpräfix auf dieser Skala eine wohldefinierte Mittelposition einnimmt - semantische Rekonstruierbarkeit -, ist nicht-regierendes mit in drei Skalenabschnitten, restituierbar', ,rekonstruierbar' und ,nichtrekonstruierbar' gleichzeitig vertreten. Ich deute das in der folgenden Tabelle durch das Symbol, $\sim$ ' an. Es verweist darauf, daß das innere Argument von mit abhängig vom Kontext restituierbar (und damit gleichzeitig rekonstruierbar) oder nur rekonstruierbar oder nicht einmal rekonstruierbar ist.

\begin{tabular}{l|c|l|l|l} 
& $\begin{array}{l}\text { vorhan- } \\
\text { den }\end{array}$ & $\begin{array}{l}\text { restituier- } \\
\text { bar }\end{array}$ & $\begin{array}{l}\text { +rekon- } \\
\text { struier- } \\
\text { bar }\end{array}$ & $\begin{array}{l}\text {-rekon- } \\
\text { struier- } \\
\text { bar }\end{array}$ \\
\hline Präposition & + & & & \\
\hline abtr. Verbpräfix & & & + & \\
\hline Adverb & & & & + \\
\hline nicht-reg. mit & & $\sim$ & $\sim$ & $\sim$
\end{tabular}

\section{Zusammenfassung}

Folgende Ergebnisse können festgehalten werden:

1. Die Präposition mit erlaubt als einzige deutsche Präposition der alten, morphologisch einfachen Schicht (im Sinne von Eisenberg 1994: 266) eine Verwendung ohne regierten Ausdruck (Nominalphrase oder Pronomen). Sie kann in dieser Verwendung nicht grundsätzlich als abtrennbares Verbpräfix eingeordnet werden, da mit bei Verbendstellung nicht in unmittelbarem Kontakt mit der Verbform auftreten muß, sondern weiter vorgerückt im Mittelfeld erscheinen kann. 
2. Das nicht-regierende mit verhält sich wie ein verb(gruppen)bezogenes Adverbiale, sowohl semantisch als auch topologisch. Damit entspricht es im Status teilweise den vergleichbaren vollständigen mit-Phrasen. Wie diese kommt das Semisatzglied subjekt- und objektbezogen vor. Diese Bezugskonstituente liefert semantisch das äußere Argument der durch mit ausgedrückten Prädikation. Ins Vorfeld verschiebbar ist das Semisatzglied jedoch nicht.

3. Die Erklärung des nicht-regierenden mit durch Ellipse der regierten Phrase erweist sich teilweise als tragfähig. So können, ähnlich wie im Hinblick auf die Valenz des Verbs, Fälle von definiter und indefiniter Ellipse unterschieden werden, wobei satzübergreifend aus Vorgängersätzen ein elidiertes Element als inneres Argument der Prädikation zu restituieren ist. Daneben gibt es Fälle satzinterner reflexiver Restitution. Die Ellipsenerklärung stößt an ihre Grenzen, weil bei dreistelligen Verben mit reflexivem Bezug (geben, schicken) das Reflexivum nicht restituierbar ist. Hier kann man nur noch von semantischer Rekonstruierbarkeit sprechen.
Noch weiter hat sich die Verwendung in Verbindung mit dem Superlativ von einer elliptischen Lösung entfernt.

4. Auch die Einordnung des nicht-regierenden mit als Adverb kann nicht vollständig befriedigen, weil Adverbien ihre inneren Argumente vollständig absorbiert haben und weder syntaktische Restitution noch semantische Rekonstruktion zulassen.

5. Nicht-regierendes mit muß daher als ein im Übergang zwischen Präposition und Adverb befindliches Element eingeordnet werden, das anders als das abtrennbare Verbpräfix keinen eindeutig bestimmbaren syntaktischsemantischen Status im Hinblick auf das innere Argument einnimmt.

Abschließend sei daran erinnert, daß sich der ambivalente Status von nicht-regierendem mit in einer orthographischen Unsicherheit widerspiegelt: Ist ein an das Verb in der rechten Klammer herangerücktes mit mit der Verbform zusammenzuschreiben oder nicht? Durch eine pauschale Kann-Regelung, wie sie in der neuen Vorlage für die amtliche Regelung 1995 vorgesehen ist, ist das Problem sicherlich nicht zu lösen.

\section{Literatur}

Bech, Gunnar ( $\left.{ }^{2} 1983\right)$ : Studien über das deutsche Verbum infinitum. Tübingen.

Becker, Karl F. ( $\left.{ }^{2} 1870\right)$ : Ausführliche deutsche Grammatik als Kommentar der Schulgrammatik. Bd. 1. Prag.

Eisenberg, Peter ( $\left.{ }^{3} 1994\right)$ : Grundriß der deutschen Grammatik. Stuttgart.

Eroms, Hans-Werner (1981): Valenz, Kasus und Präpositionen. Untersuchungen zur Syntax und Semantik präpositionaler Konstruktionen in der deutschen Gegenwartssprache. Heidelberg.

Klein, Wolfgang (1993): Ellipse. In: J. Jacobs / W. Sternefeld/A. von Stechow (Hg.), Syntax. Ein internationales Handbuch zeitgenössischer Forschung. 1. Halbband. Berlin u. a., 763-799.
Pasch, Renate (1995): Ellipse. Manuskript. Institut für deutsche Sprache Mannheim.

Primus, Beatrice (1987): Grammatische Hierarchien. Eine Beschreibung und Erklärung von Regularitäten des Deutschen ohne grammatische Relationen. München.

Sæbø, Kjell J. (1984): Über fakultative Valenz. In: Deutsche Sprache 12, 97-109.

Wilder, Chris (1996): V2-Effekte: Wortstellungen und Ellipsen. In: E. Lang / G. Zifonun ( $\mathrm{Hg}$.), Deutsch typologisch. Jahrbuch 1995 des Instituts für deutsche Sprache. Berlin / New York.

Zifonun, Gisela/Hoffmann, Ludger/Strecker, Bruno u. a. (1996): Grammatik der deutschen Sprache. Berlin / New York. 